IdeAs

Idées d'Amériques

5 | 2015

Crise ou transformations du monde du travail dans les Amériques

\title{
Le modèle d'emploi étasunien en état de crise
}

The American Employment Model in a State of Crisis

O modelo de emprego de Estados Unidos em um estado de crise

Donna Kesselman

C OpenEdition

Journals

Édition électronique

URL : https://journals.openedition.org/ideas/1004

DOI : 10.4000/ideas. 1004

ISSN : 1950-5701

Éditeur

Institut des Amériques

Référence électronique

Donna Kesselman, « Le modèle d'emploi étasunien en état de crise », IdeAs [En ligne], 5 | 2015, mis en ligne le 18 juin 2015, consulté le 20 octobre 2022. URL : http://journals.openedition.org/ideas/1004 DOI : https://doi.org/10.4000/ideas.1004

Ce document a été généré automatiquement le 20 octobre 2022.

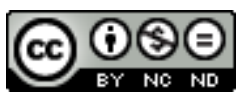

Creative Commons - Attribution - Pas d'Utilisation Commerciale - Pas de Modification 4.0 International - CC BY-NC-ND 4.0

https://creativecommons.org/licenses/by-nc-nd/4.0/ 


\title{
Le modèle d'emploi étasunien en état de crise
}

\author{
The American Employment Model in a State of Crisis \\ o modelo de emprego de Estados Unidos em um estado de crise
}

\section{Donna Kesselman}

\section{Introduction}

1 "L'Amérique est peut-être le mieux placé des pays riches en période de prospérité, mais l'Europe s'est montrée plus à même de faire face à la récession », écrivait The Economist fin $2009^{1}$. Le taux de chômage avait doublé aux États-Unis depuis le début de la crise pour atteindre 10,2\%, tandis que l'augmentation n'avait été que d'un tiers dans la zone euro. C'est le cas en Allemagne, notait la revue britannique; la production industrielle y avait pourtant plus baissé qu'outre-Atlantique. Fallait-il alors alterner les politiques publiques selon la conjoncture : tantôt la flexibilité étasunienne, tantôt les prétendues rigidités européennes, autrement dit la protection sociale? Ou ne faudraitil pas y voir plutôt une crise des modèles ? L'ambiguïté se concentre dans l'analyse de la relation de causalité. Car pour cette publication, dont le parti pris néolibéral est explicite, comme pour l'OCDE à partir des années 1980 et l'Union européenne une décennie plus tard, c'est l'existence d'un marché d'emploi flexible, incarné dans la «machine étasunienne à créer des emplois », qui représente non pas une alternative adaptée aux périodes fastes, mais le moteur même de la prospérité (Klau F., Mittelstädt A., 1986).

2 Ce modèle se caractérise par l'employment-at-will, c'est-à-dire la prérogative patronale dans l'embauche et surtout le licenciement, ainsi que par l'ancrage des avantages sociaux dans la relation contractuelle. Certes, il existe des protections publiques, mais elles sont minimales et fragmentées : leur insuffisance ne fait, en dernière analyse, que renforcer l'importance particulière aux États-Unis de la relation d'emploi comme mécanisme de redistribution des richesses sous forme d'avantages sociaux (Kesselman D., 2007). Ces deux caractéristiques correspondent aux traits majeurs de la crise sociale 
qui débute en 2008. Tout d'abord, la flexibilité de l'emploi a favorisé la croissance du chômage à un taux cinq fois plus élevé que dans la zone euro. Ensuite, les conséquences de la perte d'emploi pour le travailleur et sa famille sont dramatiques : affaiblissement de la protection sociale, et surtout perte de la couverture médicale.

Ces caractéristiques renvoient justement aux deux plans marquants du premier mandat du nouveau président arrivé au pouvoir en temps de crise: l'un sur la relance économique - notamment par la création d'emplois -, l'autre sur la réforme de l'assurance maladie. Nous allons voir à quel point ces deux dispositifs, bien que présentés de manière distincte, sont liés par l'enjeu que représente pour le pouvoir la relation d'emploi, contrairement à ce qu'on a l'habitude de penser à propos des ÉtatsUnis.

4 Nous commencerons par un état des lieux du marché du travail aux États-Unis en analysant ce qui le place en situation de "crise », donc difficilement maîtrisable, voire insaisissable. Car la crise s'exprime non seulement dans les conséquences socioéconomiques qu'elle engendre mais également sur le terrain institutionnel, comme l'atteste l'incapacité des outils statistiques à l'apprécier pleinement. On s'intéressera ensuite aux plans mis en œuvre pour faire face à cette situation. On conclura sur le sort du modèle de la «machine américaine à créer de l'emploi » dans un marché de travail international «en voie de globalisation » (Azaïs C., 2010) car c'est ce modèle même de l'emploi qui est mis à mal.

\section{État des lieux}

\section{Mesurer la crise de l'emploi}

5 L'ampleur de cette crise entraîne, pratiquement partout, des reculs du produit intérieur brut (PIB) avec des ajustements vers le bas de la condition salariale (Gautié J., 2010). Elle se traduit aux États-Unis, dans le vécu comme dans la prise de conscience, par des centaines de milliers de pertes d'emploi par mois, ajoutant, en décembre 2007, 8,2 millions de chômeurs depuis le début officiel de la récession, et 15,7 millions en octobre 2009. Le chômage, à son pic à cette date (10,2\%), n'atteint pas les $10,9 \%$ de 1981-82 ; il est toutefois à son niveau le plus élevé depuis 1983. Un tiers des chômeurs (35,6 \%) sont des chômeurs de longue durée, dépassant la période de droit à l'assurance fédérale de 26 semaines, niveau jamais vu depuis la grande Dépression (BLS, 2009a, Table A_9).

6 Les taux officiels de chômage correspondent à la définition étroite du Bureau International du Travail (BIT), qui en exclut tout travailleur ayant effectué au minimum une heure de travail (sic) au cours de la semaine de référence, ou n'étant pas à la recherche active d'un emploi ou enfin n'étant pas disponible dans les quinze jours. Les taux de chômage augmentent pendant la période, malgré la réduction de la population active, qui a baissé de 190000 personnes au mois d'octobre 2009 (BLS 2009a, Table A-1). C'est pour tenter d'améliorer ses instruments de mesure que le Bureau of Labor Statistics fédéral (BLS) ajoute désormais la catégorie des "sous-employés", comprenant les individus « marginalement attachés » à la population active. En octobre 2009, ils étaient 2,4 millions : il s'agit d'individus découragés et ayant renoncé à la recherche d'un emploi (deux fois plus que l'année précédente), ou ayant cherché un emploi depuis 12 mois, même si ceux-ci n'ont pas cherché d'emploi pendant les 4 semaines précédant 
l'enquête (dite "période de référence »). En les additionnant aux chômeurs caractérisés, cette catégorie représente $17 \%$ de la population active (BLS, 2009a, Table A-13).

7 Il faut y ajouter 9,3 millions d'individus au chômage partiel imposé et les travailleurs à temps partiel ne réussissant pas à trouver un temps plein (BLS, 2009a, Table A-5). Au total, pour ce qui est des ménages, $25 \%$ ont connu le chômage pendant la période des deux ans étudiée, et $44 \%$ ont été touchés par la perte d'emploi ou la baisse des salaires d'un des membres, selon l'Economic Policy Institute (EPI), proche des syndicats, dans ce qu'on appelle dorénavant la "Grande récession » (Great Recession).

Cet état des lieux nous permet de mieux apprécier la profondeur de la crise structurelle du marché du travail entamée juste avant l'éclatement de la bulle financière en septembre 2008, qui n'a fait qu'aggraver cette dernière et qui s'est traduite par la précarisation de la relation d'emploi. Les premiers touchés par la récession travaillaient sur des postes précaires lors des licenciements massifs d'intérimaires 44000 en moyenne par mois entre janvier 2008 et juillet 2009 (BLS, 2009a). Ces postes constituaient déjà un tiers de l'emploi dans l'industrie manufacturière; la crise a débouché sur la «création" de nouveaux postes précaires, précarisés par le temps partiel subi et la réduction des heures travaillées (Sauviat C., 2009).

9 Les moyennes nationales, à leur plus haut niveau en octobre 2009 atteignant $10 \%$, suivi de près en novembre et décembre avec $9 \%$, gomment de nombreuses disparités, tout d'abord géographiques : certains États subissant des taux de chômage bien plus élevés. Le Dakota du Nord et du Sud et le Nebraska connaissaient, en décembre 2009, des taux inférieurs à $5 \%$, le Michigan en revanche, $14 \%$. Si 33 États sur 50 affichaient des taux inférieurs à la moyenne nationale, l'un d'eux, le Wyoming, a vu le sien presque doubler, passant de $4 \%$ début 2009 à $7,5 \%$ en octobre de la même année. L'étude détaillée tend à contredire la thèse de la flexibilité comme facteur déterminant de la création ou du maintien de l'emploi. Si parmi les plus touchés se retrouvent des États fortement syndicalisés, où le licenciement est donc plus difficile et le marché du travail plus « rigide » du fait de contrats collectifs protecteurs (le Michigan, la Californie, le Rhode Island), on y compte aussi la Caroline du nord ( $11,2 \%$ de chômage) et la Caroline du sud $(12,6 \%)$, États ayant les taux de syndicalisation les plus bas des " Right-to-Work States », emblématiques du régime flexible d'employment-at-will (BLS, 2009b) ${ }^{2}$.

Les disparités sont encore plus fortes entre les sexes et les groupes ethniques. Le chômage s'est plus accentué chez les hommes que chez les femmes; en octobre 2009, le taux dans la population blanche était de $9,5 \%$, mais de $10,7 \%$ pour les hommes blancs. C'est un indice pertinent de crise qui révèle la destruction d'emplois parmi ceux les mieux rémunérés et dotés d'avantages sociaux, comme dans l'industrie manufacturière qui a perdu 2,1 millions d'emplois de décembre 2007 à octobre 2009 (BLS, 2009a, A-1, B-3). Les travailleurs hispaniques et noirs connaissaient, en octobre 2009, un taux national de chômage de $13,1 \%$ et de $15,7 \%$ respectivement (BLS, 2010). L'écart entre Blancs et Noirs était du simple au triple en Louisiane. Dans le Connecticut les Hispaniques avaient 2,5 fois plus de chance d'être au chômage que les Blancs nonhispaniques (Algernon, 2009). Le taux de chômage des Noirs dans le Michigan (19,5 \% en juillet 2009) était plus de deux fois plus élevé que dans le Rhode Island (7,6\%). À l'intérieur des États, les disparités se creusent davantage, comme dans des comtés à majorité noire en Caroline du sud. 
11 L'inégalité ethno-raciale est partie intégrante du « modèle » étasunien et la crise remet directement en cause le processus de promotion sociale des minorités, notamment noires. La loi pour les droits civiques de 1964 (Civil Rights Act) s'était en effet donné cet objectif grâce à l'accès à l'emploi. Or, presque deux générations plus tard, au moment où l'on proclame un consensus large sur l'égalité des chances, la logique insidieuse du travail précaire et les licenciements en masse minent les acquis du mouvement des droits civiques, acquis fondés sur l'emploi (Kesselman D., 2011). Et malgré certains progrès, les revenus des Noirs en 2004 n'étaient qu'un peu plus de la moitié en moyenne $(55,6 \%)$ de ceux des Blancs ; une famille noire moyenne ne pouvait capitaliser que $19 \%$ des richesses (wealth) d'une famille blanche, notamment par l'acquisition d'une maison. Depuis, la perspective d'accès à la propriété s'est encore dégradée : les minorités ont été plus frappées par les saisies, dues aux pertes d'emploi massives et à la crise des subprimes, ainsi que par des événements tragiques comme l'inondation des quartiers populaires de la Nouvelle-Orléans.

Peut-on dissocier l'existence aux États-Unis de poches de précarisation sociale du problème d'accès à l'emploi permanent? Si la population précarisée présente le même profil que dans d'autres pays industrialisés - femmes, jeunes, moins diplômés, immigrés - ce qui est propre aux États-Unis est l'extrême stratification liée à l'existence de ghettos ethniques et raciaux, surtout noirs (Wacquant, 2006). Selon une commission d'enquête du Sénat, si le taux de chômage national n'atteignait avant la récession en 2007 que $4,5 \%$, celui d'une couche particulière, celle des hommes noirs des quartiers défavorisés ne disposant pas de diplôme d'enseignement secondaire, était déjà passé de $59 \%$ à $72 \%$ en quelques années. Le chiffre était de $29 \%$ pour les Blancs et de $19 \%$ pour les Hispaniques. Pour le sénateur Charles Schumer, le sous-emploi chez les hommes noirs représentait déjà à cet époque un problème "profond, persistant et qui laisse perplexe» (Mishel L., 2007).

13 Ainsi, la crise de l'emploi doit être cernée par des données de différentes natures; elle s'exprime aussi dans l'incapacité des statistiques à évaluer son étendue, tout autant que dans des conséquences sociales échappant aux institutions de protection sociale. Il s'agit d'une crise «structurelle» du marché du travail et de l'emploi qui doit s'apprécier dans des termes « nouveaux ».

\section{Les modifications structurelles du marché du travail}

14 Des mutations structurelles, en cours depuis les années 1970, ont influé sur la notion jadis acquise de "crises cycliques ». Pour certains économistes de la Réserve Fédérale de Kansas City, trois catégories de récessions sont désormais à concevoir. La crise déclenchée en 2008 est exceptionnelle en ce qu'elle réunit les aspects les plus négatifs des deux premières (Knotek E.S., Terry S., 2009).

La récession de 1973-74 et la suivante de 1981-82 furent très sévères en termes de perte d'emploi et de ralentissement économique : les baisses respectives de 3,1\% et de $2,5 \%$ du PIB dépassaient les moyennes d'après-guerre. Elles débouchèrent pourtant sur une reprise rapide. La récession suivante de 1991-93 puis celle de 2001 furent moins sévères mais suivies de reprises plus faibles, premières illustrations de la "reprise sans création d'emplois» (jobless recovery). Même après le rétablissement de l'activité économique, le chômage n'a pas seulement stagné, il a continué à s'aggraver. 
La dernière "Grande récession » s'est traduite par une baisse d'activité sévère suivie d'une reprise, sans qu'on ait pu constater, à la fin 2010, de véritables créations d'emplois. En septembre 2009, après 21 mois consécutifs de perte d'emplois, il s'agissait de la plus longue période de perte d'emplois depuis 70 ans, dépassant de loin la moyenne d'après-guerre de 11 mois (Feldstein M., 2001 ; Shierholz H., 2009). Étant donné l'augmentation de la population, il aurait fallu créer 11 millions d'emplois, soit 582000 par mois, pour revenir au niveau d'avant la récession (Mishel L., 2009). En mars 2015 , le taux de chômage était en baisse à $5,5 \%$ avec une prévision de croissance relativement élevée par rapport aux autres grands pays, au Nord comme au Sud. Or, la création d'emplois mensuelle aux États-Unis ne représente que le tiers de ce qui serait nécessaire pour revenir au niveau d'avant la récession, et depuis un an le nombre de chômeurs n'a pas baissé. Autrement dit, si le taux de chômage a baissé depuis mai 2008 -le pourcentage d'adultes à la recherche d'un emploi est de $62,8 \%$, au plus bas depuis 37 ans -, c'est en raison de la diminution de la population active dû au retrait des demandeurs d'emplois découragés (BLS, 2015).

17 Comment expliquer cette gravité ? La récession, qui s'ajoute à la crise bancaire, y est pour beaucoup. Il n'en reste pas moins que le constat d'une « mutation structurelle » du marché du travail, avec la multiplication d'emplois de type précaire, avait déjà été fait au début du siècle par Ben Bernanke. Pour le président de la FED de l'époque, les deux tiers des emplois créés pendant la « reprise sans création d'emplois » après la récession de 2001 étaient dans cette catégorie. Il en est de même depuis la "reprise» d'octobre 2009, déclarée par le Bureau national de recherche économique (National Bureau of Economic Research, NBER 2009), chargé d'évaluer la conjoncture. La plupart des emplois créés jusqu'en 2009 l'ont été dans les services temporaires à la personne : 44000 emplois par mois depuis juillet 2008 (BLS, 2009a). Les chercheurs de la Réserve fédérale de Kansas City n'étaient alors guère optimistes pour l'emploi :

Même si les données ne permettent pas de conclure, de tels changements dans les marchés du travail depuis le début des années 1980 semblent avoir créé des conditions favorisant une réduction et un retardement des créations d'emplois dans la période qui suit une récession. Après les deux précédentes récessions, ce faible taux des créations d'emplois a contribué à des « reprises sans créations d'emplois ». Dans la mesure où ces transformations structurelles dans les marchés du travail se sont poursuivies durant la récession en cours, il est fort possible que la baisse à venir du chômage ne soit pas aussi rapide qu'à la suite d'autres récessions graves (Knotek E.S., Terry S., 2009 : 13) 3 .

18 Le président du NBER, Martin Feldstein, directeur du Council of Economic Advisors sous Reagan, mettait en garde les « optimistes » : plutôt qu'une reprise fondamentale de la croissance, les indicateurs de 2009 ne reflètaient que des dépenses temporaires de consommation sous l'effet du plan de relance (Feldstein, 2009). Les relances ultérieures, dont la dernière annoncée en septembre 2010, n'ont fait que conforter l'hypothèse de fragilité persistante de l'économie étasunienne. L'économiste de centre-gauche («liberal») Paul Krugman regrette le montant insuffisant de dépenses publiques destinées à raviver l'activité et qui est arrivé peut-être déjà trop tard (Krugman P., 2010). Dans un ouvrage, Robert Reich, ministre du Travail sous Clinton, prévoyait, quant à lui, que la «Grande récession » durerait longtemps, ôtant toute perspective de prospérité à la jeune génération et accroissant les inégalités dans la répartition de la richesse (Reich R., 2010). 
19 La crise est donc venue accélérer des mutations fondamentales en cours, dont deux concernent l'emploi. La première consiste au recours quasi-systématique à des licenciements permanents. Lors des premières récessions citées, les travailleurs faisaient l'objet en général de mises-à-pied conjoncturelles, et pouvaient s'attendre à retrouver, lors de la reprise, leur poste de travail grâce aux garanties contractuelles négociées par les syndicats. Depuis, des licenciements de masse ont abouti au démantèlement de pans entiers de l'économie productive et à l'élimination des emplois les mieux protégés, affaiblissant par là-même les bases du syndicalisme étasunien (Farber H., Western B., 2001).

20 La deuxième modification concerne la réembauche éventuelle sur un support ayant luimême changé de nature. L'organisation de la production à flux tendu recourt davantage à des configurations de mise au travail "non standards»: heures supplémentaires, travailleurs à temps partiel ou à durée déterminée, sous-traitance et externalisation. Cette flexibilité permet aux entreprises de coller au plus près aux fluctuations de la demande.

21 Ces deux tendances ont pour effet de retarder, après une récession, l'embauche sur des contrats à durée indéterminée (Aaronson D. et al, 2004). Le recours aux licenciements permanents, au lieu de mises à pied temporaires, influe sur l'évolution du chômage en période de reprise et sur ses temporalités. Le chômeur s'embarque dans un processus de recherche d'emploi, pouvant changer de branche ou de région géographique, voire passer par le détour d'une formation, sans garantie de retrouver une place. Le travail non standard retarde, au mieux, la réembauche sur la base d'un contrat stable.

22 Le temps partiel évolue aussi dans le sens de la précarisation. Une dimension statistique a été introduite par le BLS pour éclairer ce phenomène. Chez les 18-64 ans, la proportion de travailleurs à temps partiel à titre principal (primary earners), par rapport à ceux travaillant pour un complément de revenu au ménage (secondary workers), s'est considérablement accrue dans les trente dernières années. La proportion de travailleurs à temps partiel à titre principal stagne depuis cette date (BLS, 2015). Ils comptent dorénavant pour $36 \%$ des travailleurs à temps partiel, un pourcentage record (Shaefer H., 2009). Rappelons que le temps partiel est plus précaire qu'en Europe, où la loi prévoit l'égalité de traitement avec le CDI quant au salaire et aux principaux avantages sociaux. L'absence de statut de salarié à plein temps aux États-Unis (employee) entraîne l'absence quasi-systématique de telles protections.

23 L'institutionnalisation des configurations précaires par l'affaiblissement du statut salarial contractuel entraîne la fragilisation du rapport de force collectif des salariés sur le marché du travail, déclare Lawrence Mishel, de l'EPI, à une commission d'enquête du Congrès (2009). Dans le cadre historique des restructurations productives engagées depuis presque deux générations, le taux de plus de $30 \%$ de la population active pouvant entrer dans une catégorie de contrat précaire ou de sous-emploi tend à confirmer l'hypothèse, aux moins aux États-Unis, d'une précarisation sociale d'ensemble (Appay B., 2005 ; Appay B., Jefferys S., 2009).

\section{La crise dans toute sa durée}

24 Ainsi, plutôt que de se limiter à la définition d'une conjoncture selon les critères des économistes - évolution du PIB sur au moins deux trimestres consécutifsl'introduction de considérations subjectives, aussi bien que des nouvelles temporalités 
du chômage, permettent une compréhension des récessions envisagées du point de vue de ceux qui les subissent. La crise du marché du travail étasunien a précédé la récession caractérisée et se poursuit depuis la reprise officielle d'octobre 2009, ayant des conséquences pour la population, des conséquences pour bon nombre d'entre elles difficilement saisissables à ce stade.

En ce sens, une étude qui fait date s'intéresse au coût à long terme d'une récession, pour le chômeur et sa famille, à partir de celle de 2001 (Irons J., 2009). La perte de revenus, souvent non récupérés, peut être suivie de la perte de biens ou d'endettement mais, dans tous les cas, laisse des traces. En matière de réemploi, $35 \%$ des salariés à temps plein s'étant trouvés au chômage n'avaient pas retrouvé d'emploi en 2003 et $13 \%$ travaillaient à temps partiel. Ceux qui avaient retrouvé un emploi à temps plein gagnaient en moyenne $13 \%$ de moins que précédemment. Au total $60 \%$ n'avaient pas retrouvé leur niveau de vie antérieur, sans compter l'absence de réparation des pertes et torts subis.

Les études sociologiques font de même apparaître des conséquences indirectes connues sous le terme anglais de scarring c'est-à-dire de "cicatrice " - qui pèsent, à court comme à long terme, sur les familles. L'impact est démontré sur les résultats scolaires des enfants ayant vécu dans un ménage frappé par le chômage, comme sur leur développement physique et psychique, surtout en cas de malnutrition en bas âge. La famille s'avère moins à même de fournir un environnement éducatif stimulant et, vu le coût des études supérieures dans ce pays, certains ont dû les retarder ou y renoncer. Des répercussions se font sentir jusqu'à la génération suivante car les enfants d'un père ayant connu le chômage pendant la période de référence gagnaient en salaires $9 \%$ de moins que des enfants de familles comparables mais n'ayant pas connu le chômage. Cette baisse significative de la consommation porte aussi préjudice au développement économique du pays.

\section{Crise du débat, crise politique}

\section{Politique publique face à la crise}

27 Une crise se définit avant tout par la difficulté du pouvoir à y faire face. La réponse de Washington à la "Grande récession » a été double : la relance économique, notamment par l'intervention publique en faveur de la création d'emplois, et la réforme du système de couverture médicale, en modifiant son ancrage traditionnel dans la relation d'emploi contractuelle assortie d'avantages sociaux. On voit à quel point le travail et son modèle se trouvent, contrairement à ce qu'on a l'habitude de croire, au cœur des préoccupations gouvernementales étasuniennes.

Le plan de relance de l'administration Obama, l'American Recovery and Reinvestment Act (ARRA) de février 2009 allouait 787 milliards de dollars dans l'objectif de «Sauver ou créer 3,5 millions d'emplois » avant fin 2010 (Council of Economic Adviors 2009). Pour les sympathisants du président, les dépenses - en termes d'infrastructures publiques, de déduction d'impôts aux petites entreprises, de subventions aux États pour maintenir les services essentiels et éviter les licenciements de fonctionnaires (enseignants, policiers), de protection des plus vulnérables (extension de l'assurance chômage, bons alimentaires) - auront réussi à restaurer la confiance, à prévenir une chute libre de 
l'économie nationale (Bartik T., Bishop J., 2009) et à réduire d'au moins 4 points la montée du chômage (Mishel L., 2009).

Chez les conservateurs des voix s'élèvent pour réprouver l'ARRA. La Heritage Foundation, un think tank conservateur, critique les catégories délibérément vagues et invérifiables : comment comptabiliser le nombre d'emplois « sauvés "? Les aides n'aboutissent pas à de véritables créations d'emplois qui ne peuvent venir que de l'investissement du secteur privé. La fondation n'y voit que des mesures cosmétiques de soutien aux industries en déclin, comme l'automobile, et surtout à leurs syndicats, liés au Parti démocrate (Riedl B., 2010).

L'autre politique marquante est la Patient Protection and Affordable Care Act de 2009 (PACA), dont le vote doit être relié à une dimension véritablement historique, bien qu'insuffisamment soulignée, des élections de 2008. Ce scrutin présidentiel retrouvait les échos d'un plébiscite des lois du New Deal et de son interventionnisme social bienveillant. Pour la première fois dans ce pays, tous les candidats, quel que soit leur bord politique, proposaient un plan national d'assurance maladie (American Heritage Foundation, 2009). Les mêmes causes avaient abouti aux mêmes effets: la crise systémique, avec ses conséquences dramatiques pour la population, appelait une solution « providentielle».

31 Ce qu'on appelle communément désormais l'Obamacare reste la législation phare de la présidence démocrate, même si sa légitimité reste toujours contestée. Après la remise en cause de sa validité constitutionnelle par des États fédérés - contestant la prérogative dont prétend se doter l'État fédéral d'obliger les citoyens eux-mêmes à se procurer une couverture médicale, et nonobstant le jugement de la Cour suprême qui a tranché en sa faveur - la nouvelle majorité républicaine dans les deux chambres du Congrès s'y oppose. Quant aux résultats, le nombre des non-assurés a été réduit de plus d'un tiers, et ceci malgré le fait qu'en début 2015 près de la moitié des États fédérés (22 sur 50) refusaient toujours d'introduire un des dispositifs majeurs, à savoir l'extension du financement médical fédéral, le Medicaid, aux plus démunis. Plus de 5 millions de personnes se trouveraient ainsi laissées sans couverture médicale, pourtant à leur portée, selon la Maison Blanche (White House, 2015). Le devenir de la loi, toujours soumis aux aléas politiques, n'est donc pas acquis.

32 Il importe avant tout ici de souligner à quel point l'esprit de la loi fédérale pour l'assurance maladie reflète une conception particulière de la relation d'emploi. Trois types de projets avaient été envisagés. Le premier aurait étendu le système contractuel traditionnel d'attribution de l'assurance maladie par l'intermédiaire de la relation salariale, avec obligation faite aux employeurs de fournir une assurance à tous les salariés ou de payer, sous menace de pénalité, une indemnité équivalente à l'État (« play or pay»). Un autre, promu par les syndicats et l'aile gauche du Parti démocrate, favoriserait à l'inverse l'intervention étatique, à la manière du système canadien. Il s'agirait soit d'un régime public universel, dit single payer, dans la droite ligne du système d'assurance publique qui existe pour les retraités, le Medicare; soit proposition de dernier recours - la possibilité pour le citoyen d'opter pour une forme de sécurité sociale publique, dite public option.

33 Le président a finalement choisi, pour la PACA de mars 2010, l'assurance individuelle obligatoire du citoyen (individual mandate) comparable, toute proportion gardée, à l'assurance automobile. Chaque individu devra se procurer une assurance, soit par son employeur, soit directement par lui-même, sous peine de sanctions financières de 
l'agence nationale des impôts, l'Internal Revenue Service. Pour les États ayant voté le dispositif donnant accès au Medicaid, une aide est prévue pour les individus les plus démunis sous forme de subvention pour la prise d'une assurance (White House, 2010).

Sur le fond, cette acquisition individuelle complique le rapport entre l'assurance maladie et la relation d'emploi, tendant à plusieurs égards à les dissocier. Or, le modèle étasunien, au nom du non-interventionniste étatique, associe les prestations sociales à la relation contractuelle d'emploi plus que dans d'autres autres pays développés (BLS, 2014). Quelles seront donc les conséquences du nouveau système pour le marché du travail ? Plusieurs hypothèses sont envisageables.

\section{Conséquences à plus long terme pour le modèle étasunien de l'emploi}

Certains craignent la perspective de rémunérations à la baisse. Aux États-Unis, l'employeur qui intègre dans l'enveloppe un plan d'assurance maladie ou d'autres avantages en nature accorde un salaire moyen moindre. La confédération AFL-CIO, par exemple, doute que l'employeur qui ne fournirait plus d'assurance augmenterait, en conséquence, le salaire de l'employé concerné. Une autre mesure, controversée au sein même du camp démocrate, est la taxation, par la loi, du salarié bénéficiant de plans d'assurances contractuelles généreuses, appelées péjorativement "Cadillac Plans ». Ce dispositif - auparavant réclamé par le candidat républicain John McCain en 2008 et devenu cheval de bataille des syndicats contre lui- pourrait remettre en cause l'argument principal de la syndicalisation, le pouvoir de négocier des contrats collectifs plus durables et favorables aux salariés (AFL-CIO, 2008-2009).

En même temps, le dispositif du PACA le plus contraignant pour les entreprises, l'employer mandate, voit son application repoussée d'année en année, notamment sous la pression d'associations patronales, comme l'US Chamber of Commerce (2015) qui le critique comme étant préjudiciable à la croissance économique et à l'embauche, en plus d'être d'une trop grande complexité. L'employer mandate oblige une entreprise disposant d'au moins 50 salariés comptabilisés en "équivalent temps-plein » (donc additionnant toutes les heures faites, y compris à temps partiel) à proposer une assurance maladie à au moins $95 \%$ des salariés embauchés à temps plein (défini comme au moins 30 heures par semaine) et à leurs dépendants jusqu'à l'âge de 26 ans, sous peine d'amende ${ }^{4}$. Les premières évaluations des conséquences, notamment par une enquête menée par l'auteure en Californie durant l'été 2013 et 2014 dans la restauration et la grande distribution, démontrent la tendance des employeurs, dans ces secteurs, à abaisser le temps de travail des salariés sous le seuil des 30 heures.

Sans recul suffisant pour évaluer les conséquences de la loi, mais cherchant à dégager les tendances en cours, le BLS a effectué une étude sur l'attribution par les entreprises d'une assurance maladie durant les premières années d'application du PACA. En l'an 2000, $64 \%$ de la population étasunienne non retraitée (sans accès à Medicare) bénéficiait encore d'une assurance maladie par l'intermédiaire de l'employeur du secteur privé (couverture plus ou moins étendue), taux qui descend à 55,1\% en 2012 (Lambrew J., 2001 ; Antos J. 2009; BLS, 2014). Entre 2003 et 2012, la période d'étude du BLS, cette tendance à la baisse se confirme, à la fois par la baisse de l'offre de couverture médicale proposée par les entreprises et par la baisse du taux d'acceptation de cette offre par les salariés (takeup rate). 
38 La réduction des offres provient de choix patronaux, surtout des PME, du recul des contrats collectifs protecteurs négociés par les syndicats, et des contrats précaires de plus en plus nombreux et le plus souvent dépourvus de prestations sociales. La diminution en pourcentage des salariés acceptant l'offre d'une couverture médicale faite par les employeurs est due à l'augmentation de la part de contribution salariale (premiums), d'une moyenne de $200 \%$ à cette période mais plus élevée encore pour les petits salaires. En 2012, dans le premier quintile des employés aux salaires les plus élevés, seuls 15,9\% acceptent l'offre d'une assurance maladie individuelle et 8,5\% d'une couverture familiale, comparé à $72,3 \%$ et 58,3\% respectivement pour les salariés $\mathrm{du}$ quatrième quintile. Dans les deux cas la proportion est en baisse par rapport à 2003. La taille de l'entreprise est l'un des facteurs, la contribution salariale étant proportionnellement plus importante dans les plus petites entreprises. Le BLS signale d'ailleurs que ces employeurs proposent des assurances moins chères mais aussi de moindre qualité en termes de couverture (BLS, 2014)5. Ces tendances entraîneraient des conséquences sur le marché du travail en raison des «décisions individuelles des travailleurs » : le choix de tel ou tel type d'emploi en fonction des avantages associés au contrat, le choix d'entrer ou de rester dans la population active, de travailler plus ou moins d'heures, de prendre sa retraite plus ou moins tardivement.

39 Ainsi la tendance statistique confirme le déplacement de la responsabilité fondamentale de l'acquisition de l'assurance maladie du salarié vers le citoyenindividu. Aussi modestes qu'ils puissent être aux États-Unis, les avantages sociaux associés à la relation salariale, intégrés dans l'enveloppe de rémunération, pèsent sur le coût du travail (Senate, 2009). Ainsi, les politiques adoptées face à la crise dans le but d'obtenir une plus grande flexibilité - que ce soit des emplois non permanents créés par des dépenses publiques d'urgence ou la réforme de l'assurance maladie - déplacent le risque social de l'employeur vers le travailleur et sa famille. Cette tendance à l'individualisation est spécifique de l'ère néolibérale, et elle s'accélère aux États-Unis comme ailleurs.

\section{Conclusion}

40 Ainsi, la conjoncture ne fait que conforter le modèle étasunien en l'incitant à davantage de flexibilité, trait qui le caractérise déjà fondamentalement. La conséquence essentielle de la « Grande récession » a été de faire ressortir la contradiction interne de ce modèle : les avantages sociaux liés au rapport contractuel s'avèrent difficilement compatibles avec les impératifs de l'emploi flexible, dans un marché du travail en cours de globalisation. Pour leur part, les conseillers économiques du président (Council of Economic Advisors) n'ont-ils pas réaffirmé, au point culminant de la crise, «la nécessité de la flexibilité du marché du travail étasunien, caractérisé par son dynamisme et la poursuite de l'évolution technologique au XXI ${ }^{e}$ siècle ${ }^{6}$ ? (Council of Economic Advisors, 2009) Or, autre contradiction, l'intervention publique fait face aux conséquences immédiates de la crise par l'introduction de plus de flexibilité, mais les conséquences vont se poursuivre car révélatrices de transformations et de bouleversements plus profonds qui sont liés, entre autres, à la flexibilisation de la relation d'emploi et son rapport contractuel.

41 En ce qui concerne l'Europe, son modèle social n'a peut-être pas dit son dernier mot, ce qui n'exclut pas pour autant qu'il puisse évoluer. Pour un chercheur de l'Institut de 
Recherches Economiques et Sociales (IRES) : «Les risques liés à la profondeur de la crise semblent avoir conduit à une sorte de trêve dans un processus de plus long terme de remise en cause de conditions de régulation offrant une certaine sécurité aux salariés ». Or, cette régulation se fait sur des bases plus fragiles: en témoigne le nombre de salariés menacés par les impératifs de la flexibilité compétitive, chômeurs ou précaires, se situant de plus en plus en « dehors de la zone d'intervention centrale des relations sociales instituées » (Hege A., 2009).

42 L'exemple étasunien s'impose donc toujours en modèle, relayé par des organismes internationaux. Le fait que l'OCDE, et la Stratégie européenne pour l'emploi (SEE), à travers ses lignes directrices, s'emparent désormais du nouvel indicateur de la santé du marché du travail, à savoir le "taux d'emploi ", vient du modèle étasunien. La focalisation sur le taux d'emploi, sans précision sur la nature contractuelle des salariés concernés, conduit à la promotion de formes de travail flexibles. L'objectif de la SEE, lors du sommet de Lisbonne de 2000, d'atteindre $70 \%$ de taux d'emploi en Europe pour 2010, notamment pour les seniors (55-64 ans) ${ }^{7}$, se calquait sur le taux étasunien qui avoisinait 67 \% (BLS, 2009a). La politique européenne de recul de l'âge de la retraite, par exemple, remise au premier plan de l'actualité en France, appelle des mesures de mise au travail des seniors - mi-temps, temps partiel, contrats d'activation - au nom du taux d'emploi. Cette politique européenne a permis de réaffirmer le modèle étasunien de la " machine à créer de l'emploi » au moment où celui-ci traverse précisément une crise aux États-Unis, car derrière le bon résultat du taux de chômage (au plus bas niveau depuis la «Grande récession ») se cache un taux d'emploi qui est le moins élevé depuis 1978 (BLS, 2010, 2015). Autrement dit, même ce marché du travail peu réglementé n'a pu éviter des pertes massives d'emplois, ne serait-ce que dans les marchés internes les plus flexibles, malgré des créations dans des secteurs polarisés - services à bas salaires d'une part, services hautement qualifiés dotés de hauts salaires d'autre part - et dans certains secteurs plutôt conjoncturels, comme l'exploitation du gaz de schiste.

Les solutions que propose l'État ne semblent pas pouvoir servir de palliatif. La seule politique novatrice qui s'est imposée semble être la loi historique pour l'assurance maladie. Il reste qu'au nom de la création hypothétique d'un droit nouveau, la conséquence première risque d'être la remise en cause du mécanisme principal de redistribution de la richesse dans ce pays. Le processus d'individualisation des prestations sociales se renforce au moment où les transformations structurelles $d u$ marché du travail et les conséquences à plus long terme de la récession tendent à la précarisation du salariat. Peut-on parler d'une avancée vers de nouvelles garanties ou d'une remise en cause des seules garanties fondées sur un rapport de forces qu'avaient acquises les travailleurs aux États-Unis, celles de l'emploi salarié syndicalisé ?

Malgré des similitudes avec le New Deal, on peut douter, au regard de l'actualité, de l'existence aujourd'hui du type de consensus ou du rapport de forces sociales et politiques, dans ce pays comme ailleurs, qui avait pu permettre une redistribution de la richesse vers le plus grand nombre. L'enjeu des interventions publiques se trouve dans la possibilité de faire face au paradoxe de la globalisation: permettre le fonctionnement des marchés financiers et le commerce mondialisé, tout en assurant la mission démocratique de l'État d'assurer le bien-être de la population. Il s'agit d'assurer la protection des droits fondamentaux des citoyens qui jusqu'alors s'était avant tout réalisée grâce à leur accès à l'emploi. Verra-t-on l'émergence de nouvelles configurations de mise au travail aux États-Unis accompagnées de l'obtention 
d'avantages sociaux sur de nouvelles bases plus individualisées et market-based? Il s'agirait alors d'une nouvelle forme de relation d'emploi, voire d'un modèle nouveau.

\section{BIBLIOGRAPHIE}

Aaronson, Daniel, Ellen Rissman, Daniel G. Sullivan, « Assessing the Jobless Recovery »,

Federal Reserve Bank of Chicago, second quarter 2004.

AFL-CIO, www.aflcio.org, page consultée régulièrement en 2008-2009.

Algernon, Austin, « Unequal Unemployment : Racial disparities in unemployment vary widely by state », Economic Policy Institute, Issue Brief n² 257, 15 juillet 2009, http://www.epi.org/ publication/ib257/, page consultée le 15 mars 2010.

American Heritage Foundation, « American Presidents and Health Reform : A Chronology », HHN Magazine, février 2009, http://www.hhnmag.com, page consultée le 15 mars 2010.

Antos, Joseph, "The Case for Real Health Care Reform », AEI Working Paper n 148, American Enterprise Institute for Public Policy Research, 2009, http://www.aei.org/wp-content/uploads/ 2011/10/20090623-Antos.pdf, page consultée le 15 mars 2010.

Appay, Béatrice, La Dictature du succès : Le paradoxe de l'autonomie contrôlée et de la précarisation, Paris, L'Harmattan, 2005.

Appay, Béatrice et Steve Jeffreys (dir.), Restructurations, précarisation, valeurs, Toulouse, Octares Editions, 2009.

Azaïs, Christian (dir.), Labour and Employment in a Globalising World : Autonomy, Collectives and Political Dilemmas, Berne, Peter Lang, 2010.

Bartik, Timothy, et John Bishop, « The Job Creation Tax Credit : Dismal projections for employment call for a quick, efficient and effective response ", EPI Briefing Paper, $\mathrm{n}^{\circ} 248$, New York, Economic Policy Institute, 20 octobre 2009.

Bureau of Labor and Statistics (BLS), «Employment Situation Summary, October 2009 », 6 novembre 2009a, http://www.bls.gov/news.release/archives/empsit_11062009.pdf, page consultée le 17 mars 2010.

BLS, « Unemployment Rates for the States », décembre 2009b, http://www.bls.gov/news.release/ archives/laus_12182009.htm, page consultée le 3 avril 2010.

« Employment and unemployment in families by race and Hispanic or Latino ethnicity », Table 1, 2010, http://www.bls.gov/news.release/archives/famee_03242011.htm, page consultee en octobre 2014.

BLS, « Trends in employment-based health insurance coverage : evidence from the National Compensation Survey ", Monthly Labor Review, octobre 2014, http://www.bls.gov/opub/mlr/2014/ article/trends-in-employment-based-health-insurance-coverage.htm, page consultée le 1 avril 2015. 
BLS, « Employment Situation Summary March 2015 », 2015, http://www.bls.gov/news.release/ empsit.nro.htm, page consultée le 1 avril 2015.

Council of Economic Advisors, «Preparing the Workers of Today for the Jobs of Tomorrow »,Executive Office of the President, juillet 2009, http://www.whitehouse.gov/ administration/eop/cea/Jobs-of-the-Future, page consultée le 15 mars 2010.

Europa, « Employment and Social Policies », Union européenne, 2010, http://eur-lex.europa.eu/ summary/chapter/employment_and_social_policy.html?root_default=SUM_1_CODED=17, page consultée le 15 mars 2010.

Farber, Henry, et Bruce Western, « Accounting for the Decline of Unions in the Private Sector, 1973-1998 », Journal of Labor Research, vol. 22, n³, septembre 2001, p. 459-485.

Feldstein M., et K. Feldstein, « From Recession to Recovery », NBER, 4 décembre 2001, http:// www.nber.org/feldstein/bg120401.html, page consultée le 15 mars 2010.

Feldstein, Martin, « Has the U.S. Recovery Begun ? ", NBER, juin 2009, http://www.nber.org/ feldstein/projectsyndicate_usrecoverybegun_june2009.html, page consultée le 15 mars 2010. Gautié, Jérôme, Entretiens de l'IRES, Paris, Institut de Recherches économiques et sociales, 11 février 2010, http://www.ires.fr, page consultée le 15 mars 2010.

Hege, Adelheid, « Les acteurs sociaux face à la crise », Chronique internationale de l'IRES, $\mathrm{n}^{\circ} 121$, novembre 2009, p. 3-5.

Irons, John, « Economic scarring : The long-term impacts of the recession », EPI Briefing Paper $n^{\circ}$ 243, 2009.

Kesselman, Donna, «Travail et salariat aux États-Unis : Quels droits, quelles perspectives ?», Revue française d'études américaines, $\mathrm{n}^{\circ}$ 111, 2007, p. 6-26.

Kesselman, Donna, « Travail précaire et précarisation institutionnelle aux États-Unis », Sociologias, 2011, p. 66-100.

Klau, Friedrich et Axel Mittelstädt, « Labor Market Flexibility », OECD, Economic Studies nº 6, 1986, http://www.oecd.org/general/searchresults/? q=labor\%20market\%20flexibility\&cx=012432601748511391518:xzeadubob0a\&cof=FORID: $11 \&$ ie=UTF-8, page consultée le 15 mars 2010.

Knotek, Edward S. II et Stephen Terry, « How Will Unemployment Fare Following the Recession? », Economic Review, Federal Reserve Bank of Kansas City, octobre 2009, https:// www.google.fr/url?

$\mathrm{sa}=\mathrm{t} \& \mathrm{rct}=\mathrm{j} \& \mathrm{q}=\&$ esrc=s\&source=web\&cd=1\&ved=0CCEQFjAAahUKEwinuLbcvZHGAhWB1xQKHYpzAAQ\&url=https\%3A\%2F\%2Fwww.kansa Vae9IoGvU4rngSA\&usg=AFQjCNH_2smYz7PbUA49vkvrrUVgX2JvsA\&sig2=ywVNyhSaMvn5wUiEy2IOuQ\&bvm=bv. 95515949,d.d24\&cad=rja, page consultée le 15 mars 2010.

Krugman, Paul, « US Spending Plan Is Too Little, and Perhaps Too Late », New York Times, 28 septembre 2010.

Lambrew, Jeanne, «How The Slowing U.S. Economy Threatens Employer-Based Health Insurance ", The Commonwealth Fund, 2001, http://www.cmwf.org, page consultée le 15 mars 2010.

Mishel, Lawrence, Jared Bernstein et Sylvia Allegretto, The State of Working America : 2006/2007, Ithaca, Cornell University Press, Economic Policy Institute, 2007.

Mishel, Lawrence, « Letter from Lawrence Mishel », EPI, 2009, http://www.epi.org, page consultée le 15 mars 2010. 
National Bureau of Economic Research (NBER), « The NBER's Business Cycle Dating Procedure », octobre 2009, http://www.nber.org/cycles/recessions_faq.html, page consultée le 15 mars 2010.

Reich, Robert, Aftershock : The Next Economy and America's Future, New York, Alfred A. Knopf, 2010.

Riedl, Brian, « White House Report Claims Stimulus Success-Despite 3,5 Million Job Losses », American Heritage Institute, 14 janvier 2010, http://www.heritage.org/research/reports/2010/01/ white-house-report-claims-stimulus-success-despite-3-million-job-losses, page consultée le 15 mars 2010.

Sauviat, Catherine, «À crise sans précédent, mesures sans précédent mais business as usual », Chronique internationale de l'IRES, $\mathrm{n}^{\circ}$ 121, 2009.

Shaefer, H. Luke, « Part-Time Workers : Some Key Differences Between Primary And Secondary Workers ", Monthly Labor Review, octobre 2009.

Senate (Congrès des Etats-Unis), « Background Materials for Senate Committee On Finance Fountable on Health Care Financing ", Joint Committee on Taxation, Report JCX-27-09, 8 mai 2009, https://www.jct.gov/publications.html?func=startdown\&id=3557, page consultée le 15 mars 2010.

Shierholz, Heidi, « Jobs Picture for October 2, 2009 », EPI, 2009, http://www.epi.org/publication/ jobs_picture_for_october_2_2009/, page consultée le 15 mars 2010.

US Chamber of Commerce, « ObamaCare Employer Mandate », http://www.uschamber.com/ health-reform/employer-mandate, page consultée le 1 avril 2015.

Wacquant, Loïc, Parias urbains : Ghetto, banlieues, État, Paris, La Découverte, 2006.

White House, « Health Reform in Action », 2010, http://www.whitehouse.gov/issues/health-care, page consultée le 1 avril 2015.

White House, « 22 States Are Refusing to Expand Medicaid. Here's What That Means for Their Residents ", 14 janvier 2015, http://www.whitehouse.gov/share/medicaid-map, page consultée le 1 avril 2015 .

\section{NOTES}

1. «America may lead the rich world in periods of prosperity, but Europe has shown a greater talent for dealing with recession", traduction de l'auteure extraite du texte original (The Economist, novembre 2009).

2. L'expression fait référence à des dispositions juridiques appelées "right-to-work statutes ", rendant la syndicalisation (et les formes de protection sociale qui l'accompagnent souvent) plus difficile d'accès dans certains États, notamment dans le Sud.

3. «While the evidence is not conclusive, such changes in labor markets since the early 1980s appear to have produced conditions conducive to restrained, delayed hiring in the aftermath of recessions. Following the most recent two recessions, this weakness in hiring contributed to jobless recoveries. To the extent that these structural changes in the labor market have persisted through the current recession, it is distinctly possible that unemployment going forward may not decline as rapidly as it did following other severe recessions ", traduction de l'auteure extraite du texte original (Knotek et Terry, 2009).

4. D'autres aspects du dispositif font toujours l'objet de tractations politiques, par exemple un taux maximum de contribution du salarié selon son niveau de revenu et un degré minimal de couverture dans l'assurance proposée par l'employeur. 
5. Si on ajoute le paramètre de la qualité de la couverture offerte, l'augmentation du coût ne fait pas monter, mais au contraire baisser, le taux d'acceptation par les salariés. Dans le secteur privé en 2012, pour une couverture médicale prévoyant les soins optiques (vision care), seuls $4,2 \%$ des salariés du premier quintile et $32,2 \%$ du quatrième quintile ont accepté, des proportions dans les deux cas en baisse par rapport à 2003.

6. «Worker flexibility is key given the dynamic nature of the US labor market and ongoing technological change ». Traduction de l'auteure extraite du texte original (Council of Economic Advisors, 2009).

7. Le taux d'emploi d'une classe d'individus est calculé en divisant le nombre d'individus ayant un emploi par le nombre total d'individus en âge de travailler, généralement de 15 à 64 ans (Europa, 2010).

\section{RÉSUMÉS}

La « machine à créer des emplois » étasunienne est présentée comme le modèle le plus adapté au marché du travail et de l'emploi globalisé du XXI ${ }^{\text {ème }}$ siècle. Quelles en sont les caractéristiques? Dans quelle mesure réussit-il à faire face aux effets de la crise? L'article analyse des modifications structurelles qui se sont affirmées depuis la crise de 2008.

The U.S. job machine has been promoted as the best adapted model to the $21^{\text {st }}$ century global labor and employment market. What is this model, precisely? How has it fared in the face of the crisis and what are its effects? The article analyses the structural changes which have become more apparent since the advent of the 2008 Great Recession".

Este verdadeiro modelo, a « máquina norte-americana de criar empregos », é apresentado como o padrão que a especialização adaptou ao mercado globalizado do $\mathrm{XXI}^{\circ}$ século. Quais são as suas características? Em que medida sofreu os efeitos da crise? O artigo analisa as modificações estruturais, que se afirmaram desde a crise de 2008.

\section{INDEX}

Keywords : work, employment, United States, model, recession, health care, Obamacare Palavras-chave : trabalho, emprego, Estados-Unidos, modelo, caída, seguro-doença, Obamacare Mots-clés : travail, emploi, États-Unis, modèle, récession, assurance maladie, Obamacare

\section{AUTEUR}

\section{DONNA KESSELMAN}

Donna Kesselman est Professeure des Universités à l'Université Paris-Est Créteil. Elle publie largement sur les questions du travail, de l'emploi et des relations professionnelles, en proposant une perspective comparative (France, États-Unis, Brésil, Canada). Elle est co-rédactrice d'un recueil d'articles publié aux Presses Universitaires de Provence, articles issus des communications qui ont été faites lors du Congrès de l'IdA de 2013 "Les Femmes dans les 
Amériques", et plus particulièrement de l'axe thématique "Les femmes et le travail dans les Amériques". Elle a co-rédigé des études de la Direction de l'animation de la recherche, des études et des statistiques (DARES) sur les relations professionnelles. Elle est au bureau du RT 18 de l'Association française de sociologie traitant des relations professionnelles. 